Original Paper http://ajol.info/index.php/ijbcs http://indexmedicus.afro.who.int

\title{
Typologie des exploitations agricoles familiales : cas de la périphérie de la forêt protégée de Baban Rafi du Niger
}

\author{
Barmo SOUKARADJI ${ }^{1 *}$, Amani ABDOU ${ }^{1}$, Sitou LAWALI ${ }^{2}$, Ichaou ABOUBACAR ${ }^{1}$, \\ Ali MAHAMANE ${ }^{3}$ et Mahamane SAADOU ${ }^{2}$ \\ ${ }^{1}$ Institut National de la Recherche Agronomique du Niger (INRAN), BP 429 Niamey, Niger. \\ ${ }^{2}$ Université Dan Diko Dankoulodo de Maradi, BP 465227 Maradi Niger, Niger. \\ ${ }^{3}$ Université de Diffa, BP 78 Diffa, Niger. \\ *Auteur correspondant ; E-mail : barmo_inran@yahoo.fr
}

\section{REMERCIEMENTS}

Les auteurs remercient l'Université Dan Dicko Dankoulodo de Maradi et l'Institut National de la Recherche Agronomique du Niger (INRAN) pour le partenariat grâce auquel ce travail de terrain a été financé.

\section{RESUME}

La gestion durable d'une formation forestière nécessite la prise en compte des activités humaines s'exerçant aux alentours en raison de leurs impacts sur la pérennité et la qualité des produits exploités. Cette étude visait ainsi à caractériser les exploitations agricoles familiales adjacentes de la forêt de Baban rafi. Pour y arriver, des enquêtes socio-économiques ont été réalisées sur un échantillon de 313 exploitants. L'analyse des données par la classification ascendante hiérarchique et le test de Kruskal Wallis ont permis d'identifier 4 groupes d'exploitations que sont les grands agriculteurs éleveurs moyens, les agriculteurs moyens petits éleveurs, les grands éleveurs petits agriculteurs et les petits agriculteur-éleveurs. Les critères pris en compte sont la superficie cultivée, les productions et revenu agricole, la couverture alimentaire, le cheptel et les modes d'acquisition des terres. Il ressort que les grands agriculteurs éleveurs moyens, contrairement aux 3 autres groupes jouissent d'une production et de revenu agricole suffisant leur garantissant une autosuffisance alimentaire. Le groupe de grands éleveurs petits agriculteurs détient le plus gros cheptel (25,7 UBT) et exerce par conséquent une forte pression pastorale (32,8 tonnes de matières sèches) sur les ressources de la forêt. Les 4 groupe, d'exploitants prélèvent annuellement 2,6 à 3 tonnes de bois.

(C) 2017 International Formulae Group. All rights reserved.

Mots clés: Exploitation agricole familiale, caractérisation, forêt, Niger.

\section{Typology of family farms: case of area of Baban Rafi protected forest of Niger}

\section{ABSTRACT}

The sustainable management of forestry vegetation requires taking into account the human activities of the area according to their impact on durability and quality of the exploited products. The purpose of this study was to characterize the family farms of area of Baban rafi forest, where socio-economic survey was carried out 
on 313 farmers. Data were analyzed with R (Software) using Kruskal Wallis test and four groups of farms were identified of which the big farmers holding medium herd, medium farmers holding small herd, big livestock farmers using small land and small farmer/livestock keepers. The criteria taken into account are the cultivated area, productions and incomes, food blanket, number of animals raised and land acquisition. It appears that the big farmers holding medium herd contrary to three other groups have good productions and incomes to ensure food self-sufficiency. The group of big livestock farmers using small land has largest herd (25.7 TLU) and therefore exerts strong pastoral pressure (32.8 tons of dry matter) on forestry resources. The four groups of farmers harvest annually 2.6 to 3 tons of wood.

(C) 2017 International Formulae Group. All rights reserved.

Keywords: Family farm, characterization, forest, Niger.

\section{INTRODUCTION}

Les exploitations agricoles familiales (EAF) jouent un rôle important dans la vie socio-économique des communautés rurales. Elles constituent la principale source de nourriture, de revenu et d'emploi pour ces communautés. L'hétérogénéité de ces exploitations agricoles longtemps considérée comme un obstacle à la modernisation rapide de l'agriculture est liée à des facteurs divers. Il s'agit entre autres des differents modes d'accès au foncier, de la diversité des ressources du milieu, de la différence d'âges et de niveau d'instruction (Jamin et al., 2007). La prise en compte de cette diversité dans les projets de développement permet d'améliorer et garantir l'efficacité et le succès des interventions auprès des producteurs. Cependant, il est plus difficile dans la pratique de tenir compte des particularités de chacune des exploitations ou, à l'opposé, de considérer toutes les exploitations agricoles comme un ensemble homogène. L'exploitation agricole familiale est caractérisée par ses facteurs de production (terre, force de travail, capital y compris le cheptel, consommations intermédiaires) avec à sa tête un chef d'exploitation qui décide (partiellement tout au moins) des modalités de la production et notamment de l'allocation des facteurs de production. Elle est également considérée comme une unité familiale de production, de consommation, d'accumulation et de résidence préoccupée principalement par la recherche de la sécurité alimentaire de la famille (Mbétid-Bessane, 2006).

En revanche, l'agriculture familiale dont la base de la main d'œuvre est essentiellement familiale permet d'organiser les productions agricole, pastorale, forestière et halieutique sous la gestion d'une famille. Durant ces dernières décennies, les exploitations agricoles connaissent des mutations du fait de la rareté des terres de cultures liée à la forte croissance démographique et de la dégradation du capital productif naturel due aux épisodes de sécheresse, aux changements du climat global et à l'exploitation abusive des ressources naturelles. Il en résulte une différenciation accrue entre les exploitations, une forte concurrence pour l'accès au foncier, une extension des superficies cultivées et une charge pastorale relativement importante (Lawali, 2011; Maidawa, 2013). Des terres parfois marginales sont ainsi mises en valeur et des espaces protégés destinés aux activités pastorales et forestières sont défrichés au profit des cultures.

La région de Maradi abritant la forêt de Baban Rafi est la plus peuplée du Niger avec une densité de plus de 105 habitants $/ \mathrm{km}^{2}$ (INS, 2012). Cette forêt est exploitée depuis 1991 à travers 22 marchés ruraux de bois, mais elle est aussi convoitée en tant que réserves de terres pour l'agriculture. L'essentiel des exploitations sont de petite taille et à faible revenu dû à l'éclatement des familles étendues et aux morcellements 
excessifs des terres. Ces exploitations exercent sur la forêt de Baban Rafi divers types de pressions dont le surpâturage, le défrichement, l'exploitation de bois et des produits forestiers non ligneux et le feu de brousse. En outre, la caractérisation des exploitations agricoles et de leurs stratégies d'exploitation des ressources naturelles, la connaissance des menaces qui pèsent sur la forêt pourraient aider les pouvoirs publics à prendre en compte les préoccupations paysannes dans l'aménagement forestier et pastoral de la forêt de Baban Rafi.

L'objectif principal de cette étude est de caractériser les exploitations agricoles familiales de la périphérie de la forêt de Baban Rafi. Les objectifs spécifiques poursuivis consistent à : décrire le profil sociodémographique des exploitations familiales; identifier les différents types d'exploitation; déterminer les différents modes d'acquisition des terres et quantifier les principales pressions s'exerçant sur la forêt.

Dans cet article, la caractérisation des exploitations agricoles de la forêt de Baban Rafi a été faite sur la base des enquêtes socioéconomiques auprès des différentes communautés vivant à la périphérie de la forêt.

\section{MATERIEL ET METHODES Zone d'étude}

L'étude a été menée dans les villages adjacents de la forêt de Baban Rafi située dans la partie méridionale du département de Madarounfa. Cette forêt est comprise entre les latitudes $13^{\circ}$ et $13^{\circ} 20^{\prime}$ Nord et les longitudes $6^{\circ} 40^{\prime}$ et $7^{\circ} 30^{\prime}$ 'Est et couvre une superficie de 35540 ha (Figure 1). Le climat de la zone est du type sahélo-soudanien avec deux saisons : une saison des pluies de juin à septembre et une saison sèche d'octobre à mai. Le diagramme ombrothermique (Figure 2) établi à partir des moyennes mensuelles de 1984 à 2015 de la station météorologique de l'aéroport de Maradi la plus proche $(50 \mathrm{~km}) \mathrm{du}$ site indique selon les critères de Bagnouls et
Gaussen (1953) que : le mois d'août est le plus pluvieux avec environ 180,08 $\mathrm{mm}$ de pluie ; une seule période humide qui s'étale de juillet à septembre ; une période sèche étendue sur 8 mois (d'octobre à mai). Cependant, lorsqu'on considère les critères d'Aubreville (1956) qui définit le mois sec comme le mois ayant reçu moins de $30 \mathrm{~mm}$ de pluie, la période humide revient à 4 mois (juin à septembre).

La population riveraine de la forêt répartie sur 73 villages et hameaux est estimée à 67730 habitants dont $51,26 \%$ de femmes et $48,74 \%$ d'hommes (INS, 2012). Les ethnies majoritaires sont les Hausa $(66 \%)$ et les Peuhls (21\%). La densité élevée de la population $\left(105,25\right.$ habitants $\left./ \mathrm{km}^{2}\right)$ constitue un indicateur de la saturation foncière et de la forte pression sur les ressources naturelles.

L'agriculture et l'élevage constituent les principales activités économiques. A celles-ci s'ajoute également l'exploitation du bois de chauffe à travers les 22 marchés ruraux de bois mis en place à la suite de l'aménagement de la forêt de Baban Rafi. Les principales espèces exploitées sont Combretum nigricans Lepr. ex Guill. et Perr., Combretum. Micranthum G. Don, Combretum glutinosum Perr. ex DC. et Guiera senegalensis J.F. Gmel. La superficie des terroirs agricoles adjacents de la forêt est estimée à 50439 ha représentant $62 \%$ des unités d'occupation de terre de la commune de Gabi et est essentiellement consacrée à la production céréalière (Mahamane et al., 2007). Le cheptel est estimé à environ 216700 têtes dont 35547 têtes de bovins, 52771 têtes d'ovins, 115060 têtes de caprins, 3128 têtes de camelins, 3220 têtes d'équins et 6981 têtes d'asins (MEL, 2015).

\section{Echantillonnage et collecte des données}

Le sondage est à deux (2) degrés, avec comme choix d'échelles d'échantillonnages emboitées où l'unité primaire est le village et l'unité secondaire l'exploitation agricole. Au premier degré, les enquêtes ont été menées dans 21 villages, soit $28,76 \%$ des 73 villages 
de la zone d'étude. Ces villages ont été sélectionnés de façon raisonnée parmi les 73 situés autour de la forêt. Les critères de choix sont la proximité de la forêt (moins de $15 \mathrm{~km}$ ), la taille du village (plus de 400 habitants) et l'accessibilité à la forêt (Figure 1). Dans chaque village, 15 exploitations agricoles ont été enquêtées suivant trois critères (diversité ethnique, genre et nature des activités socioprofessionnelles). $\mathrm{Au}$ total, 313 exploitations agricoles ont été enquêtées.

Deux outils de collecte de données ont été utilisés : un guide d'entretien semi structuré et un questionnaire individuel, $\mathrm{Au}$ cours des entretiens semi structurés organisés en focus group, les informations collectées ont concerné la situation géographique, la démographie, les équipements et infrastructures, les modes de gestion de pâturage, les organisations sociales/associations et projets intervenant ou ayant intervenu dans le village. Quant aux enquêtes individuelles, elles ont permis de recueillir les informations relatives aux: caractéristiques sociodémographiques de l'exploitation, activités agricoles, pastorales et forestières, activités extra-agricoles, transactions des produits agricoles, superficies de terres emblavées et leurs modes d'acquisition.

\section{Analyse des données}

Les données ont été analysées au moyen des logiciels SPSS 20 et R (R development Core Team., 2010). Une analyse descriptive a d'abord été réalisée pour déterminer les principales caractéristiques de position (moyenne, fréquence, maximum, minimum) et de dispersion (écart type). Ensuite, une classification ascendante hiérarchique (CAH) a été appliquée aux données pour effectuer le regroupement des exploitations enquêtées en types aussi homogènes que possibles, à l'aide des variables qui les discriminent au mieux. Ces variables retenues sont les unes agronomiques (superficie cultivée par exploitation, ration de superficie par individu, production agricole, cheptel bovin, cheptel petits ruminants, revenu agricole, pression pastorale, pression de forestière et couverture alimentaire) et les autres sociodémographiques (personnes à charge et l'âge du chef d'exploitation).

La matrice types-variables des groupes issus de la CAH a été soumise à une analyse factorielle discriminante en vue d'identifier les variables qui discriminent lesdits groupes. Un test non paramétrique, le test de Kruskal Wallis a été réalisé pour comparer les moyennes des groupes. Pour faciliter la comparaison des revenus tirés des exploitations dont les spéculations sont différentes, les productions ont été converties en valeur monétaire à partir d'un prix moyen diffusé par le système d'information sur les marchés du Niger (SIM). Le cheptel vif des bovins et petits ruminants a été converti en Unité de Bétail Tropical (UBT), équivalent à un bovin de référence de $250 \mathrm{~kg}$ de poids sur la base de $: 1$ bovin $=1$ UBT $; 1$ ovin adulte $=$ 1 caprin adulte $=0,2$ UBT $; 1$ asin $=0,4$ UBT et 1 camelin $=1,2$ UBT (Ministère de la Coopération, 1998). La pression pastorale a été calculée sur la base qu'une UBT de $250 \mathrm{~kg}$ peut consommer $6,25 \mathrm{~kg}$ de matière sèche par jour (Boudet, 1984) en fonction des jours de pâturage dans la forêt. La pression forestière a été estimée à partir de la quantité de bois énergie utilisée par personne et par jour en milieu rurale $(0,8 \mathrm{~kg} /$ personne/jour $)$ pendant une période d'exploitation donnée (CIRAD et al., 2015).

Enfin pour la nomination des groupes, il a été utilisé le vocable "éleveur» pour ressortir le poids de l'élevage bien que ce dernier soit un élément de l'agriculture au sens large du terme. 


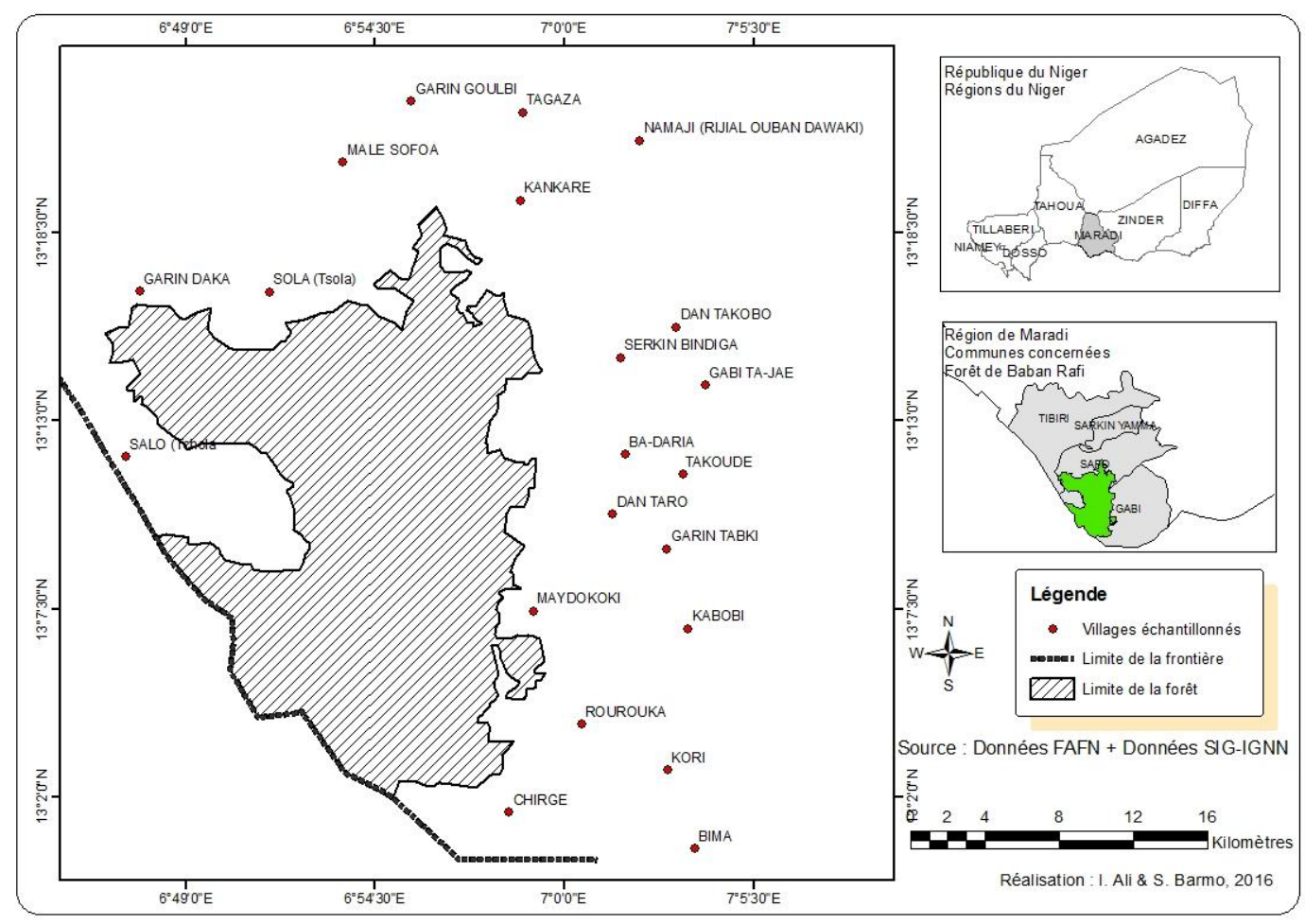

Figure 1: Localisation de la forêt de Baban Rafi et répartition des villages périphériques échantillonnés.

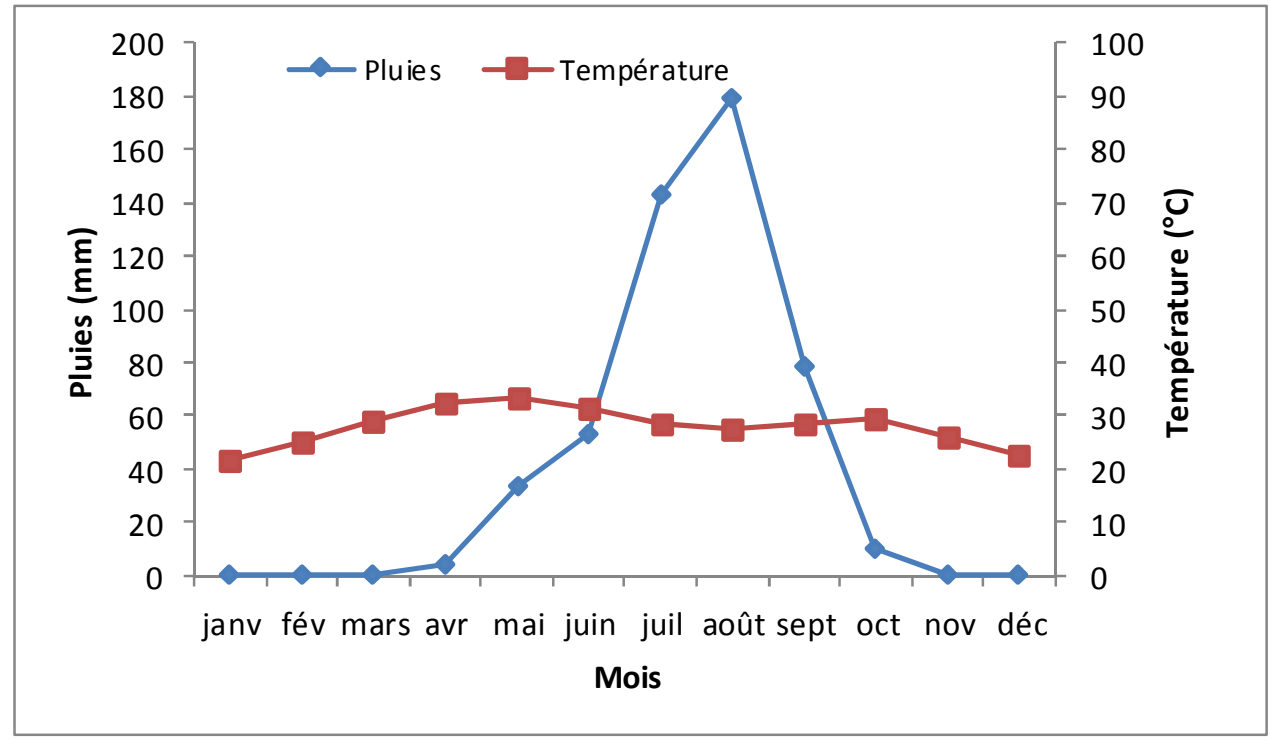

Figure 2 : Diagramme ombrothermique de la station météorologique de l'aéroport de Maradi de 1984 à 2015. 


\section{RESULTATS}

\section{Analyse socioéconomique des exploitations agricoles}

Trois cent treize (313) exploitations agricoles familiales constituées de $68,37 \%$ dirigées par des hommes et $31,63 \%$ dirigées par des femmes ont été enquêtées. Leur âge varie de 20 à 90 ans avec une moyenne de $45,70 \pm 12,51$ ans (Tableau1). La tranche de 40 à 50 ans avec une occurrence de $33 \%$ constitue la classe modale. Environs $81 \%$ des chefs d'exploitation ont un âge compris entre 30 et 60 ans. Les plus jeunes chefs d'exploitation (de 20 à 30 ans) représentent $10 \%$ et les plus vieux (plus de 60 ans) représentent environ $8 \%$. Ces exploitations comportent en moyenne $11,60 \pm 5,27$ personnes à charge avec un minimum de 3 personnes et un maximum de 34 personnes.

La proportion des chefs d'exploitation (CE) ayant reçu l'instruction coranique traditionnelle est environ 36\% alors que 20\% ont reçu l'éducation formelle (primaire et secondaire). En ce qui concerne l'alphabétisation, $20 \%$ des $\mathrm{CE}$ en ont bénéficié (Tableau 1).

La superficie moyenne des champs exploitée est de $3,23 \pm 3,52$ ha avec un minimum de 0,5 ha et un maximum de 30 ha. Ces champs sont acquis de plusieurs modes parmi lesquels l'héritage $(45,05 \%$ des exploitations), l'achat $(27,80 \%)$ et le don $(21,09 \%)$ sont les plus répandus. La location, le prêt et le gage sont peu pratiqués (Tableau1).

La production agricole varie de 99,50 $\mathrm{kg}$ à $15060,00 \mathrm{~kg}$.

\section{Typologie des exploitations agricoles}

Il est ressorti de l'application de la $\mathrm{CAH}$ à la matrice de 313 exploitations croisées aux 11 variables plusieurs partitions possibles. La partition issue du dendrogramme a été retenue en raison de l'équilibre qu'elle présente dans l'effectif des différents groupes obtenus. Cette partition met en évidence 4 groupes d'exploitations agricoles familiales autour de la forêt de Baban Rafi (Figure 3).

La classification ascendante hiérarchique $(\mathrm{CAH})$ sur les 11 variables a montré que les deux premiers axes expliquent $93,4 \%$ des informations relatives à la structure des exploitations agricoles périphériques de la forêt de Baban Rafi. En outre, chacune des valeurs de Lambda de Wilks associée aux 2 premiers axes est inférieur à 0,5 indiquant que la discrimination des groupes est bien faite (Tableau 2). Ces deux axes sont alors suffisants pour identifier les caractéristiques les plus pertinentes pouvant discriminer les différents groupes d'exploitation (Figure 4). Quatre groupes d'exploitation se distinguent : le groupe 1 (G1), positionné dans la partie négative de l'axe 1 et positive de l'axe 2 , se discrimine par une superficie cultivée importante, une production et un revenu agricoles élevés et une bonne couverture alimentaire. Cette catégorie d'exploitants est identifiée comme étant les grands agriculteurs et éleveurs moyens; le groupe 3 (G3) se trouvant dans la partie négative des axes 1 et 2 regroupe les exploitants disposant d'un cheptel bovin et petits ruminants important et exerçant par conséquent une pression pastorale forte sur la forêt. Il s'agit de grands éleveurs petits agriculteurs; le groupe 4 (G4) situé dans la partie positive des axes 1 et 2 est caractérisé par des faibles valeurs de superficie, de production et revenu agricoles et de couverture alimentaire. Ce groupe est constitué de petits agriculteur-éleveurs; enfin le groupe 2 (G2) est identifié comme le 
groupe des agriculteurs moyens petits éleveurs. Ils sont caractérisés par des valeurs moyennes de superficies cultivées, de production et de revenu agricoles et de couverture alimentaire. Leurs cheptels sont très faibles, d'où une pression pastorale subséquemment très faible sur la forêt. En considérant comme seuil une corrélation de $50 \%$, l'analyse discriminante indique que l'axe 1 est corrélée négativement à la superficie cultivée et donc à la production agricole, à la couverture alimentaire et au revenu agricole. Quant à l'axe 2, il est négativement corrélé aux variables cheptel bovin et petit ruminant et subséquemment à la pression pastorale sur la forêt (Tableau 3).

\section{Caractérisation des exploitations agricoles}

Le groupe 1 (G1) est composé des producteurs ayant des grandes superficies agricoles $(7,24 \pm 4,78$ ha) et pratiquant moyennement l'élevage (1,84 à 2,08 UBT). Ce groupe qui représente $1 / 4$ de l'effectif des exploitants enquêtés jouit d'un bon niveau d'instruction [instruction coranique $(30,12 \%)$, d'alphabétisation $(30,12 \%)$ et niveau primaire et secondaire $(20,48 \%)]$. Cette catégorie de producteurs occupe la première place en termes de production agricole, de revenu agricole, de superficie cultivée, de ratio superficie par personne, et de couverture alimentaire (Tableau 4).

Le groupe $2(\mathrm{G} 2)$, représentant 27,5\% des enquêtés, est constitué des producteurs ayant de superficie moyenne $(2,37 \pm 0,91 \mathrm{ha})$ et pratiquant peu d'élevage $(0,94$ à 1,11 UBT). Comme le précédent, ce groupe a un bon niveau d'instruction. On dénombre $36,05 \%$ d'instruits coraniques, $15,12 \%$ d'alphabétisés et $30,23 \%$ de niveau primaire et secondaire (Tableau 5). Ces producteurs occupent la deuxième position sur le plan de production agricole, de revenu de superficie cultivée et de couverture alimentaire.

Quant au groupe $3(\mathrm{G} 3)$, représentant $11,18 \%$ des enquêtés, il est formé des d'exploitants pourvus d'important cheptel $(9,34$ à 16,35 UBT) et de superficies agricoles faibles $(1,80 \pm 0,90 \mathrm{ha})$. Ce groupe se particularise par sa forte pression pastorale et forestière sur la forêt. S'agissant du niveau d'instruction, ce groupe comporte le plus fort pourcentage d'analphabètes (40\%). En effet, on note que $37,14 \%$ d'individus ont l'instruction coranique, $20 \%$ le cours d'alphabétisation et 3\% seulement le niveau primaire et/ou secondaire (Tableau 5). Ce groupe occupe le troisième rang en termes de production agricole, de revenu, de superficie cultivée et de couverture alimentaire.

Enfin, le groupe 4 (G4) représentant $34,8 \%$ d'enquêtés, il est constitué d'exploitations familiales disposant de petites superficies agricoles $(1,31 \pm 0,65$ ha) et de faible cheptel $(0,14$ à 0,73 UBT $)$. On dénombre dans ce groupe $39,45 \%$ d'individus ayant l'instruction coranique, 17,43\% d'alphabétisés et $17,43 \%$ d'individus ayant le niveau primaire et/ou secondaire (Tableau 5). En termes de production agricole, de revenu, de superficie emblavée et de couverture alimentaire, cette catégorie de producteurs est en quatrième position.

Les modes d'acquisition des terres, variables en fonction des groupes d'exploitation, évoluent de 26 à $51 \%$ pour l'achat, 41 à $51 \%$ pour l'héritage, 4 à $36 \%$ pour le don, 1 à $4 \%$ pour la location, le prêt et le gage (Figure 4). 
Tableau 1 : Caractéristiques socio-économiques générales des exploitations agricoles familiales.

\begin{tabular}{|c|c|c|c|}
\hline Paramètres & Minimum & Moyenne & Maximum \\
\hline Age de l'exploitant (an) & 20,00 & $45,7 \pm 12,51$ & 90,00 \\
\hline Nombre de personnes à charge & 3,00 & $11,6 \pm 5,27$ & 34,00 \\
\hline Superficie cultivée (ha) & 0,50 & $3,23 \pm 5,52$ & 30,00 \\
\hline Ration de superficie par individu (ha) & 0,03 & $0,31 \pm 0,31$ & 2,33 \\
\hline Production agricole $(\mathrm{kg})$ & 99,50 & $1502,64 \pm 1773,74$ & 15060,00 \\
\hline Couverture besoins alimentaires (mois) & 2,24 & $5,14 \pm 3,24$ & 24,18 \\
\hline Revenu agricole (x1000 F CFA) & 24,60 & $2173,37 \pm 3182,31$ & 56338,83 \\
\hline Cheptel bovin (UBT) & 0,00 & $2,69 \pm 8,52$ & 100,00 \\
\hline Petits ruminants (UBT) & 0,40 & $2,09 \pm 4,00$ & 34,40 \\
\hline \multicolumn{4}{|l|}{ Niveau d'instruction (\%) } \\
\hline Aucun & - & 23,64 & - \\
\hline Primaire et secondaire & - & 20,13 & - \\
\hline Alphabétisation & - & 20,45 & - \\
\hline Coranique & - & 35,78 & - \\
\hline \multicolumn{4}{|l|}{ Modes d'acquisition de terre (\%) } \\
\hline Achat- & - & 27,8 & - \\
\hline Don & - & 21,09 & - \\
\hline Gage & - & 1,6 & - \\
\hline Héritage & - & 45,05 & - \\
\hline Location & - & 2,56 & - \\
\hline Prêt & - & 1,92 & - \\
\hline
\end{tabular}




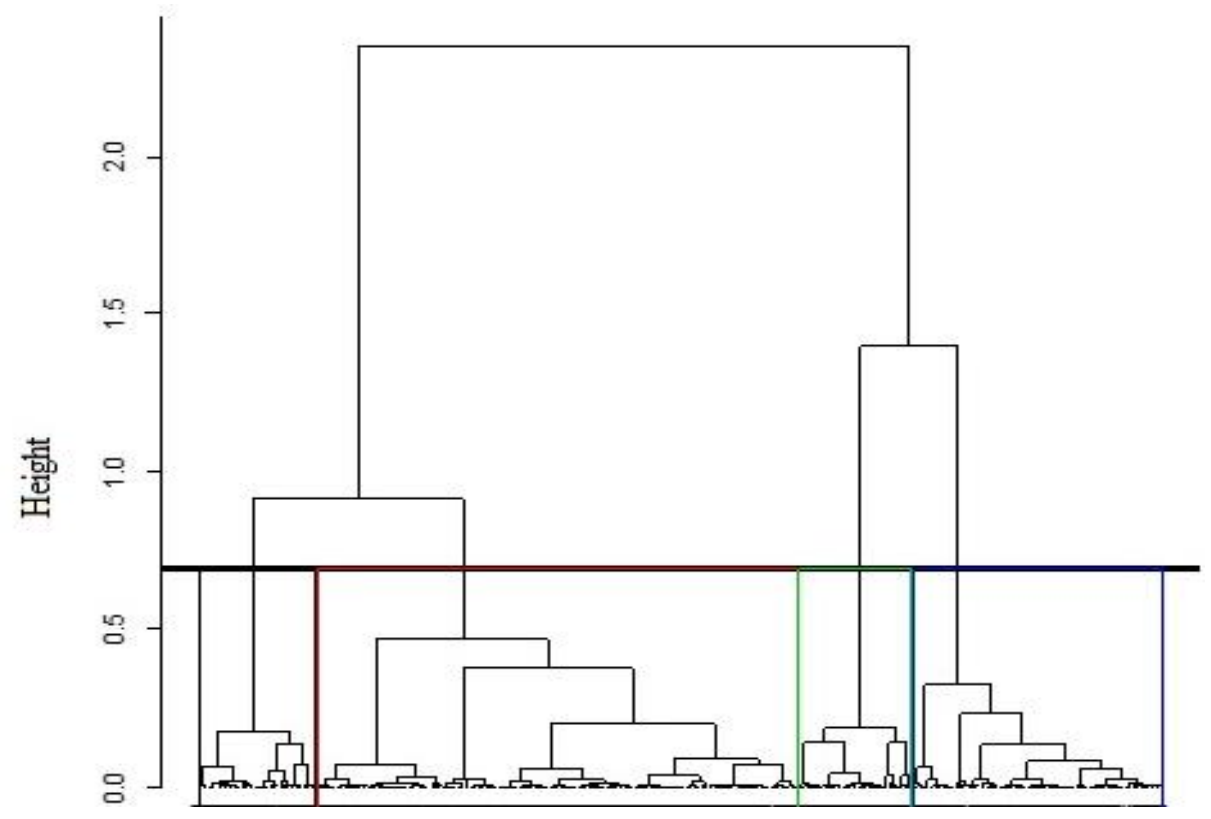

Figure 3 : Dendrogramme des exploitations agricoles familiales de la zone adjacente de la forêt de Baban Rafi.

Tableau 2 : Analyse discriminante sur les caractéristiques socio-économiques.

\begin{tabular}{|c|c|c|c|c|}
\hline Axes & Valeurs propres & Lambda de Wilks & Proportions & P-Values \\
\hline 1 & 4,67 & 0,04 & 63,66 & $<2,2 \mathrm{e}-16^{* * *}$ \\
\hline 2 & 2,18 & 0,21 & 29,77 & $<2,2 \mathrm{e}-16^{* * *}$ \\
\hline 3 & 0,48 & 0,67 & 6,57 & $<2,2 \mathrm{e}-16^{* * *}$ \\
\hline
\end{tabular}

Tableau 3 : Corrélation entre les variables et les deux premiers axes du plan factoriel de la CAH.

\begin{tabular}{lcc}
\hline & Axe 1 & Axe 2 \\
\hline Variables & Coefficients de corrélation (\%) \\
\hline Age (an) & $-9,65$ & $-5,67$ \\
Charge ménage (nombre de personnes) & $-2,73$ & $-5,33$ \\
Superficie cultivées (ha) & $-91,75$ & 21,59 \\
Ratio superficie/individu (ha/individu) & $-73,83$ & 21,99 \\
Production agricole (kg) & $-88,29$ & 11,79 \\
Couverture alimentaire (mois) & $-82,41$ & 22,10 \\
Revenu agricole (F CFA) & $-78,35$ & 13,25 \\
Pression pastorale (tonne de MS) & $-47,72$ & $-79,58$ \\
Pression bois de chauffe (kg) & $-2,29$ & $-9,01$ \\
Cheptel bovin (UBT) & $-39,96$ & $-83,54$ \\
Cheptel petit ruminant (UBT) & $-24,12$ & $-73,31$ \\
\hline MS =
\end{tabular}

MS = matière sèche $;$ UBT $=$ Unité de Bétail Tropical 
B. SOUKARADJI et al. / Int. J. Biol. Chem. Sci. 11(3): 1096-1112, 2017

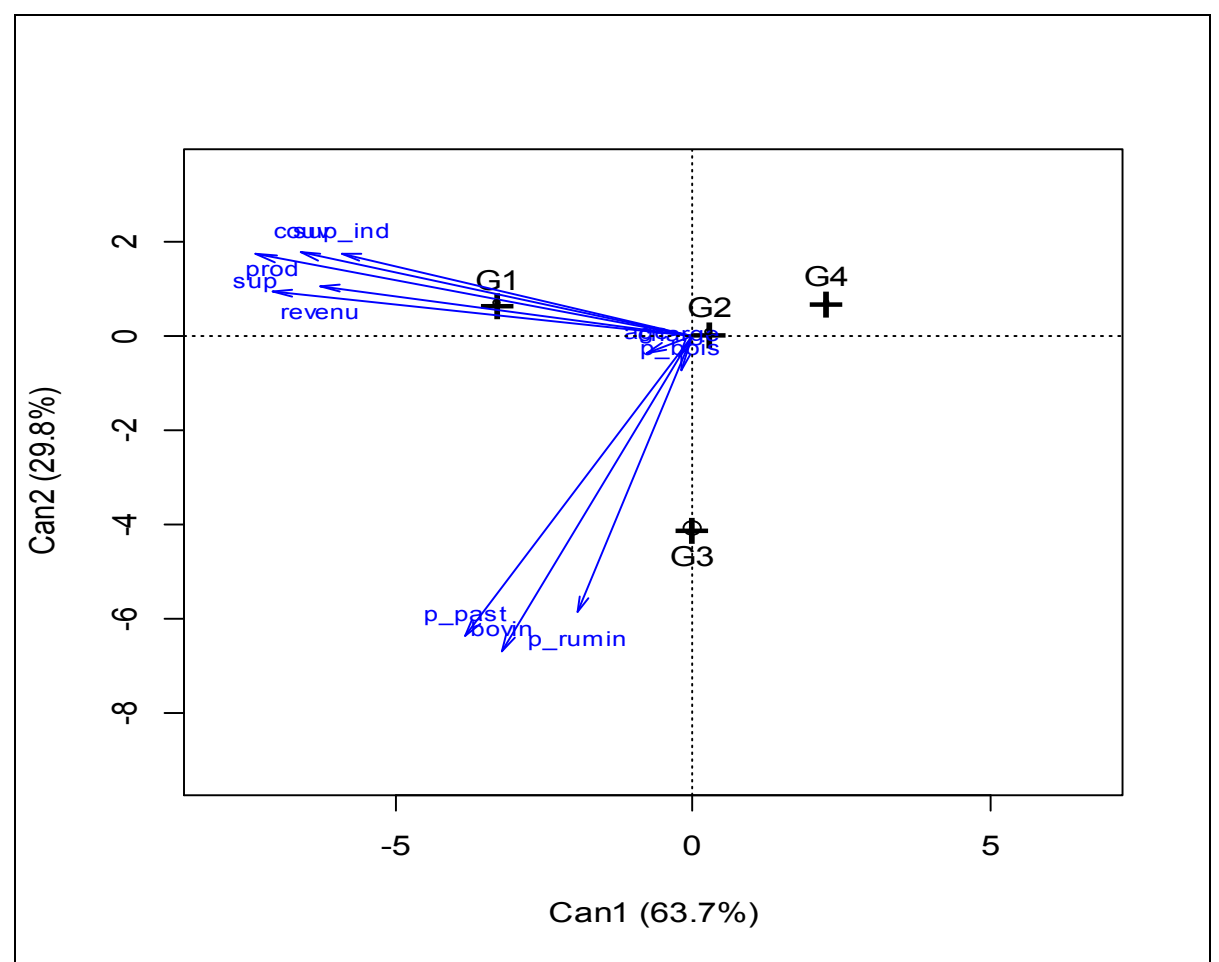

Figure 4 : Distribution des quatre groupes d'exploitation agricole dans un système d'axes discriminants. G1 : Groupe1 ; G2 : Groupe2 ; G3 : Groupe3 ; G4 : Groupe4 ; sup :

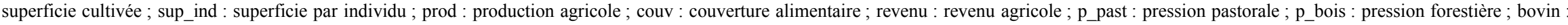
cheptel en bovin ; p_rumin : cheptel en petits ruminants 
Tableau 4 : Principales caractéristiques des exploitations agricoles riveraines de la forêt protégée de Baban Rafi.

\begin{tabular}{|c|c|c|c|c|c|}
\hline Caractéristiques & Groupe $1:(25,52 \%)$ & $\begin{array}{c}\text { Groupe } 2: \\
(27,48 \%)\end{array}$ & $\begin{array}{c}\text { Groupe } 3: \\
(11,18 \%)\end{array}$ & $\begin{array}{c}\text { Groupe } 4 \text { : } \\
(34,82 \%)\end{array}$ & P-value \\
\hline $\begin{array}{l}\text { Superficie moyenne des } \\
\text { champs (ha) }\end{array}$ & $7,24 \pm 4,78 \mathrm{a}$ & $2,37 \pm 0,91 b$ & $1,80 \pm 0,90 \mathrm{c}$ & $1,31 \pm 0,65 d$ & $<0,0001$ \\
\hline $\begin{array}{l}\text { Ratio de superficie par } \\
\text { individu }\end{array}$ & $0,66 \pm 0,41 \mathrm{a}$ & $0,30 \pm 0,15 b$ & $0,15 \pm 0,09 \mathrm{c}$ & $0,12 \pm 0,05 \mathrm{c}$ & $<0,0001$ \\
\hline $\begin{array}{l}\text { Production agricole } \\
\text { moyenne }(\mathrm{kg})\end{array}$ & $3512,05 \pm 2405,61 \mathrm{a}$ & $1090,55 \pm 408,33 b$ & $784,19 \pm 451,84 \mathrm{c}$ & $528,37 \pm 368,82 \mathrm{~d}$ & $<0,0001$ \\
\hline $\begin{array}{l}\text { Cheptel moyen en } \\
\text { bovins (UBT) }\end{array}$ & $2,08 \pm 1,81 b$ & $0,94 \pm 1,01 \mathrm{c}$ & $16,35 \pm 20,82 a$ & $0,14 \pm 0,52 \mathrm{~d}$ & $<0,0001$ \\
\hline $\begin{array}{l}\text { Cheptel moyen petits } \\
\text { ruminants (UBT) }\end{array}$ & $1,84 \pm 1,84 b$ & $1,11 \pm 0,98 \mathrm{c}$ & $9,34 \pm 8,55 a$ & $0,73 \pm 0,44 d$ & $<0,0001$ \\
\hline $\begin{array}{l}\text { Revenu annuel moyen } \\
\text { (x1000 Fcfa) }\end{array}$ & $7657,04 \pm 6174,9 a$ & $274,01 \pm 105,3 b$ & $196,02 \pm 114,0 \mathrm{c}$ & $131,24 \pm 90,6 \mathrm{~d}$ & $<0,0001$ \\
\hline $\begin{array}{l}\text { Pression pastorale } \\
\text { moyenne (tonnes) }\end{array}$ & $5,263 \pm 4,288 b$ & $2,657 \pm 1,977 \mathrm{c}$ & $32,840 \pm 38,361 \mathrm{a}$ & $1,099 \pm 1,047 \mathrm{~d}$ & $<0,0001$ \\
\hline $\begin{array}{l}\text { Pression forestière } \\
\text { moyenne (tonnes) }\end{array}$ & $2,730 \pm 1,205 a$ & $2,027 \pm 0,949 b$ & $2,950 \pm 1,412 \mathrm{a}$ & $2,572 \pm 1,006 \mathrm{a}$ & $<0,0001$ \\
\hline $\begin{array}{l}\text { Couverture alimentaire } \\
\text { (mois) }\end{array}$ & $8,744 \pm 4,133 a$ & $4,930 \pm 1,365 b$ & $3,512 \pm 0,974 c$ & $3,075 \pm 0,592 \mathrm{~d}$ & $<0,0001$ \\
\hline $\begin{array}{l}\text { Nombre moyen des } \\
\text { personnes à charge }\end{array}$ & $12,634 \pm 5,580 \mathrm{a}$ & $9,383 \pm 4,393 b$ & $13,657 \pm 6,539 a$ & $11,908 \pm 4,660 \mathrm{a}$ & $<0,0001$ \\
\hline $\begin{array}{l}\text { Age moyen des chefs } \\
\text { d'exploitation (ans) }\end{array}$ & $47,012 \pm 11,752 \mathrm{a}$ & $43,616 \pm 11,474 a$ & $48,114 \pm 12,300 \mathrm{a}$ & $45,578 \pm 13,767 a$ & 0,195 \\
\hline
\end{tabular}

UBT= Unité de Bétail Tropical

Les moyennes suivies de la même lettre sur une même ligne ne sont pas statistiquement différentes.

Tableau 5 : Niveau d'instruction par groupe d'exploitations agricoles familiales de la périphérie de la forêt de Baban Rafi.

\begin{tabular}{lcccc}
\hline Instruction & Groupe 1 & Groupe 2 & Groupe 3 & Groupe 4 \\
\hline Aucun (\%) & 19,28 & 18,60 & 40,00 & 25,69 \\
Primaire + Secondaire (\%) & 20,48 & 30,23 & 2,86 & 17,43 \\
Ecole coranique (\%) & 30,12 & 36,05 & 37,14 & 39,45 \\
Alphabétisation fonctionnelle & 30,12 & 0,15 & 20,00 & 17,43 \\
$(\%)$ & & & & \\
\hline
\end{tabular}




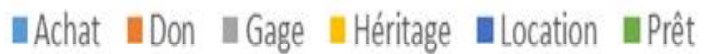

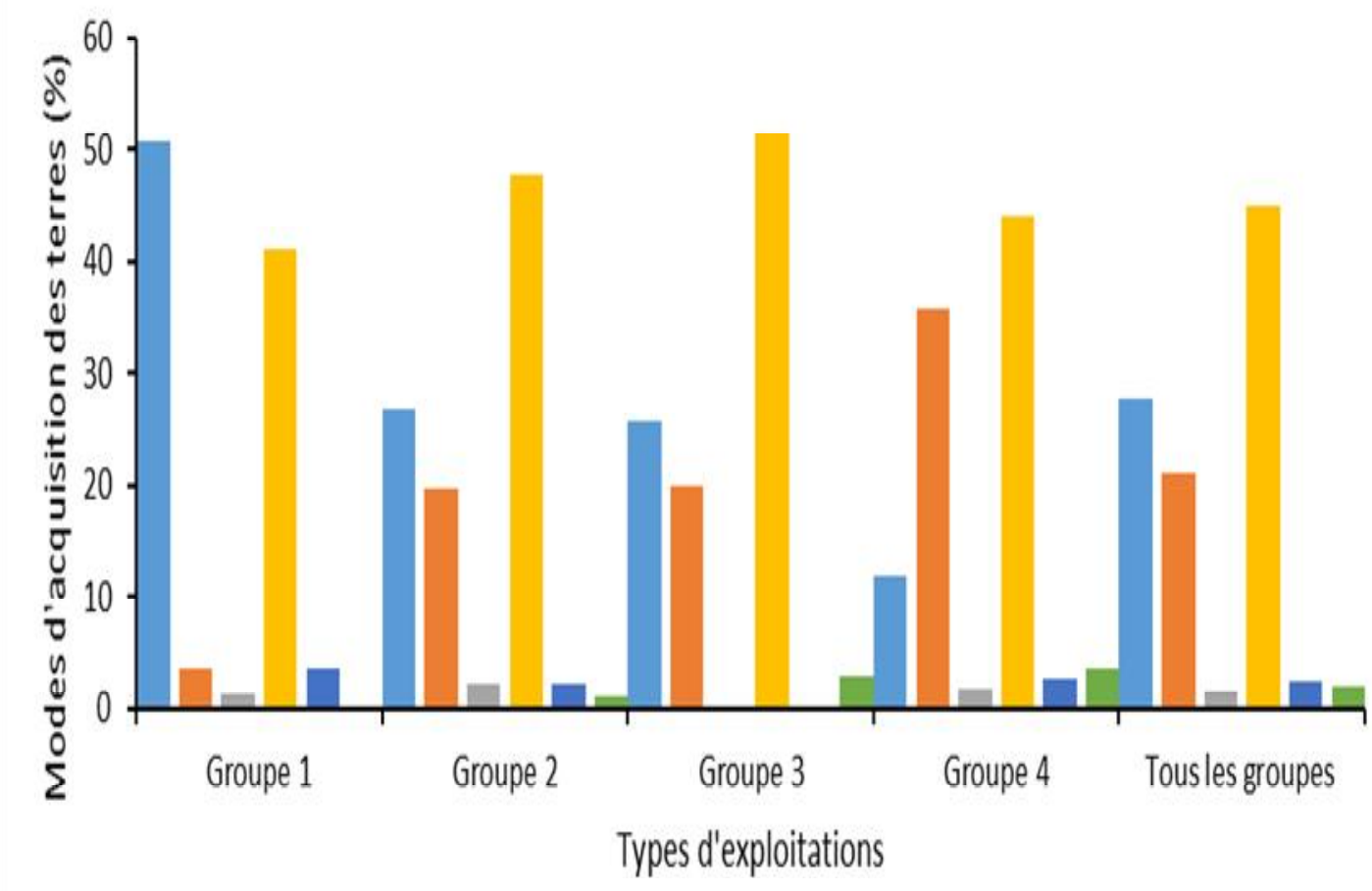

Figure 5 : Modes d'acquisition des terres par groupe d'exploitation de la périphérie de la forêt de Baban Rafi.

\section{DISCUSSION}

Quatre groupes d'exploitations agricoles familiales ont été identifiés dans les terroirs villageois de la périphérie de la forêt protégée de Baban Rafi, sur la base des 11 variables agronomiques et sociodémographiques : ce sont le groupe des grands agriculteurs éleveurs moyens (G1), le groupe des agriculteurs moyens petits éleveurs (G2), le groupe des grands éleveurs petits agriculteurs (G3) et celui des petits agriculteur-éleveurs (G4). Cette typologie révèle aussi le caractère mixte de l'exploitation agricole à travers l'intégration agriculture-élevage qui se retrouve dans tous les groupes et la prépondérance des petites exploitations matérialisées par les groupes G2, G3 et G4 qui représentent environ $73 \%$ des exploitations. Cette intégration agricultureélevage ne doit pas cacher une troisième composante sous-jacente qui est l'activité forestière. En effet, la forêt de Baban Rafi constitue par excellence la zone de pâturage et d'exploitation de bois de chauffe, de service et des produits forestiers non ligneux.

$$
\text { L'intégration agriculture-élevage- }
$$
foresterie peut être perçue comme une stratégie paysanne d'adaptation et/ou 
d'atténuation face aux risques socioenvironnementaux. En effet, cette intégration permet aux paysans de diversifier les activités, de réduire les risques comme l'insécurité alimentaire et d'améliorer la fertilité des sols (Coly et al., 2011). La prépondérance des petites exploitations reflète la caractéristique générale de l'agriculture au Niger, souvent annoncée par plusieurs auteurs (Lawali, 2011 ; Eric et al., 2012).

L'ordre décroissant d'importance des 4 groupes d'exploitation (G1, G2, G3 et G4) est corrélé positivement à la superficie, à la production agricole, au revenu et à la couverture alimentaire. Les exploitants du groupe G1 disposent de grandes superficies de terre de l'ordre de 7 ha représentant plus de 7 fois celle de G4 et 3 fois celles de G2 ou G3. Ces exploitants ont acquis leurs terres par achat $(51 \%)$ et par héritage (41\%). Par contre, au niveau du groupe G2, les modes d'acquisition dominants de terre sont l'héritage (48\%) et l'achat $(27 \%)$. La même logique est observée chez le groupe G3 avec l'héritage (51\%) et l'achat (26\%). Enfin, pour le groupe $\mathrm{G} 4$, le don occupe la deuxième place $(36 \%)$ après l'héritage $(44 \%)$. Il ressort que l'héritage, l'achat et le don demeurent les principaux modes d'acquisition de terres traduisant ainsi l'évolution des pratiques socio-économiques et culturelles de la populations rurale.

La prédominance de l'héritage sur les autres modes s'explique par le fait que la gestion de terres reste encore lignagère. Cette transmission se fait généralement de père en fils selon le principe islamique. En effet, en islam, la terre se transmet par héritage suivant les proportions de $2 / 3$ pour le garçon et $1 / 3$ pour la fille (Lawali, 2011).
L'acquisition de terre par achat est une pratique récente au Niger (Cochand, 2007). Elle est très développée dans la région de Maradi après les grandes sécheresses de 1983 et 1984 et du fait de la forte pression démographique que connaît actuellement la région (Jouve, 2007 ; Lawali, 2011). Avec la monétarisation des terres, ce sont généralement les grands exploitants au bon pouvoir d'achat qui acquièrent ces terres mais pour leur mise en valeur, ils sont obligés d'avoir recours à la main d'œuvre extérieure. En effet, les exploitants qui détiennent de faibles superficies agricoles ont tendance à les cultiver en peu de temps et travailler à l'extérieur de leur exploitation pour gagner un peu d'argent à travers le salariat agricole. Ces stratégies sont largement répandues dans l'ensemble des systèmes mixtes de production en Afrique (Eric et al., 2012 ; Lawali, 2011) et dans le monde (Herrero et al., 2010). L'achat de terre permet aux exploitants de pérenniser et sécuriser le foncier. L'intensification du phénomène de monétarisation de la terre engendrera des paysans sans terre comme dans le cas des exploitations agricoles familiales du périmètre irrigué de l'office du Niger au Mali (Coulibaly et al., 2006). En effet, ce dénuement progressif des paysans en terres couplé à une croissance démographique élevée constituent une menace potentielle sur la forêt qui pourrait faire l'objet de défrichement frauduleux.

S'agissant du mode d'acquisition par le don, il se fait sans réellement de contrepartie (monétaire ou en nature) sur la base des relations familiales et de mariage (un beaupère à son beau-fils) mais aussi du maintien des bonnes relations au sein de la communauté. Cela dénote jusqu'à présent le 
fort lien familial et de solidarité en milieu rural souligné par Lawali (2011) et Rueff (2011).

Les 4 groupes d'exploitations ne se discriminent pas seulement par le foncier ou le mode d'acquisition. En effet, il existe une différence significative entre ces groupes en ce qui concerne la production agricole, le revenu et la couverture des besoins alimentaires. Le groupe G1 avec une production moyenne de 3,5 tonnes et un revenu annuel de l'ordre de 7657000 FCFA est la seule catégorie d'exploitants autosuffisante toute l'année (couverture alimentaire 8,7 \pm 4 mois). Outre qu'ils jouissent d'un bon pouvoir d'achat et d'un capital foncier, ces grands exploitants sont généralement plus ouverts à l'utilisation des nouvelles technologies qui permettent le maintien et l'amélioration de la productivité agricole (FAO, 2011).

En revanche, la production agricole des petits exploitants (environ 1 tonne pour G2, 0,8 tonne pour G3 et 0,5 tonne pour G4) ne leur permet pas de couvrir les besoins alimentaires. Pour subvenir à ces besoins, ils sont obligés de mener des activités extra agricoles comme le petit commerce, l'artisanat, le bucheronnage, l'exode saisonnier et autres activités dans le village ou hors village. Ces constats corroborent ceux de Lawali (2011) au Niger, Akouehou et al. (2013) au Benin, Bélières (2014) au Mali et Jamin et al. (2007) en Afrique de l'Ouest et du Centre sur les petits exploitants. Ces auteurs constatent que les petits exploitants ont recours aux activités extra agricoles pour pallier l'insuffisance de la production agricole.
Sur le plan pastoral, tous les groupes d'exploitations pratiquent l'élevage mais à des degrés divers comme l'a souligné aussi Agossou et al. (2015) dans la basse vallée de l'Ouémé au Benin. En prenant uniquement la variable cheptel (bovin et petits ruminants), l'ordre de classement décroissant des groupes d'exploitations devient G3, G1, G2 et G4. Il apparaît donc que les exploitations du groupe G3 ayant le plus gros cheptel (bovins et petits ruminants) de 25,7 UBT exercent, par conséquent, la plus forte pression (32,8 tonnes de matière sèche) sur les ressources de la forêt. Si cette pression n'est pas réglementée, elle peut avoir des impacts négatifs sur la forêt car il est unanimement reconnu que l'action du bétail (pâture, broutage et piétinement) provoque des modifications dans les milieux fréquentés par les animaux (Gaucherand, 2005; Massouroudini et al., 2016). A cette pression pastorale s'ajoute la pression due au prélèvement $\mathrm{du}$ bois de chauffe, du bois de service et des produits forestiers non ligneux. En effet, tous les 4 groupes d'exploitation en fonction de leur charge en personnes prélèvent de quantité non négligeable de bois dans la forêt. Les prélèvements les plus élevés sont ceux de G1 (2,7 tonnes), G3 (3 tonnes) et G4 (2,6 tonnes) qui ont une taille statistiquement identique (12 à 14 personnes). Ces prélèvements qui sont appelés à augmenter avec la croissance démographique et l'augmentation $\mathrm{du}$ cheptel doivent attirer l'attention des gestionnaires.

\section{Conclusion}

Les exploitations agricoles familiales périphériques de la forêt Baban Rafi ont une taille moyenne d'environ 12 personnes et sont majoritairement dirigées par les hommes 
d'âge variant de 20 à 90 ans et dont le niveau global d'instruction est relativement bon. Quatre principaux groupes sont identifiés : le groupe des grands agriculteurs éleveurs moyens (G1) dont les modes d'acquisition des terres sont l'achat (51\%) et l'héritage (41\%); le groupe des agriculteurs moyens petits éleveurs (G2) et le groupe des grands éleveurs petits agriculteurs (G3) qui acquièrent les terres par achat $(48 \%$ à $51 \%)$ et l'héritage (27\%). Enfin, le groupe des petits agriculteuréleveurs (G4) au sein duquel l'héritage (44\%) et le don (36\%) sont les modes d'acquisition des terres les plus dominants. Il ressort que tous les exploitants pratiquent l'élevage à des degrés variables pour pallier les déficits agricoles récurrents et exercent par conséquent de fortes pressions pastorale et forestière sur la forêt, ce qui pourrait, à long terme, provoquer la déforestation et, en conséquence, la perte de la diversité végétale et animale de cet écosystème. Ainsi, la connaissance des caractéristiques de chaque groupe d'exploitations défini constitue un gage de la réussite de toute politique de gestion durable de la forêt de Baban Rafi. En conséquence, tout aménagement durable de cette forêt doit tenir compte des activités agrosylvopastorales de la population riveraine. Cette exigence est impérieuse dans la mesure où l'aménagement vise à stopper le défrichement de la forêt et à réguler le flux du bétail à la capacité de charge du milieu.

\section{CONFLIT D'INTERETS}

Les auteurs de ce manuscrit déclarent qu'il n'y a aucun conflit d'intérêt entre eux.

\section{CONTRIBUTIONS DES AUTEURS}

Dans la réalisation de la présente étude, BS et AA ont élaboré le protocole de recherche, collecté et traité les données et aussi rédigé le manuscrit. SL et IA ont participé à la relecture du document; en outre SL a supervisé les enquêtes socioéconomiques. AM et MS ont apporté des conseils dans la collecte et le traitement des données. Ils ont tous approuvé la version finale du manuscrit.

\section{REFERENCES}

Agossou G, Gbehounou G, Zahm F, Agbossou EK. 2015. Typologie des exploitations agricoles de la basse vallée de l'Ouémé, République du Benin. Agron. Afri., 27(3): 285-300. DOI : https://www.ajol.info/index.php/aga/artic le/view/130414/119995

Akouehou GS, Houndonougbo A, Tente B. 2013. La dynamique des systèmes de production dans les terroirs agricoles riverains de la forêt intercommunale de Fita-Agbado dans les communes de Dassa- Zoumé et de Savalou, Département des Collines au Centre du Bénin. Int. J. Biol. Chem. Sci., 7(5): 1877-1891.

DOI : http://dx.doi.org/10.4314/ijbcs.v7i5.8

Aubreville A. 1956. Conception moderne en bioclimatologie et classification des formations végétales. Bois et Forêts de Tropiques, 47: 297-306.

Bagnouls F, Gaussen F. 1953. Période de sécheresse et de végétation C.R.A.C. SC, CC XXX VI 10. 1075- 1077.

Bélières JF. 2014. Agriculture familiale et politiques publiques au Mali. Document ART-Dev : Mali ; 35. 
Boudet G. 1984. Manuel sur les Pâturages Tropicaux et les Cultures Fourragères $\left(4^{\text {ème }}\right.$ édn). Manuels et Précis d'Elevage IEMVT ; 266p.

CIRAD, BEIE, CFTC. 2015. Programme National des Energies Domestiques du Niger, rapport final, $187 \mathrm{p}$.

Cochand J. 2007. La petite irrigation privée dans le sud Niger : potentiels et contraintes d'une dynamique locale, Le cas du sud du Département de Gaya, Mémoire de Master, Université de Lausanne, $149 \mathrm{p}$.

Coly I, Diome F, Dacosta H, Malou R, Akpo LE. 2011. Typologie des exploitations agropastorales du terroir de la NEMA (Sénégal, West Africa). Int. J. Biol. Chem. Sci., 5(5): 1941-1959. DOI : https://www.ajol.info/index.php/ijbcs/art icle/view/77132

Coulibaly Y, Bélières JF, Koné Y. 2006. Les exploitations agricoles familiales $\mathrm{du}$ grand périmètre irrigué de l'Office du Niger au Mali : évolutions et perspectives. Cah. Agric., 15(6): 562569. DOI: http://dx.doi.org/10.1684/ agr.2006.0024

Eric V, Mahamoudou K, Mélanie B, Kalifa C, Mohamadoun AD, Nadine A. 2012. Intégration Agriculture-Elevage et Intensification Ecologique dans les Systèmes Agrosylvopastoraux de l'Ouest du Burkina Faso, Province du Tuy in Partenariat, Modélisation, Expérimentations : quelles Leçons pour la Conception de l'Innovation et l'Intensification Ecologique ? Cirad: Bobo-Dioulasso, Burkina Faso ; 13p.

Food and Agriculture Organization (FAO). 2011. La Situation Mondiale de
l'Alimentation et de l'Agriculture 20102011. Le Rôle des Femmes dans l'Agriculture: Combler le Fossé entre les Hommes et les Femmes pour Soutenir le Développement. FAO: Rome; 174. (http://www.fao.org/docrep/013/i2050f/i 2050f.pdf)

Gaucherand S. 2005. Effets des pratiques pastorales sur la biodiversité et sa dynamique dans les pelouses des alpages des alpes du nord : apport des traits fonctionnels des plantes. Thèse de doctorat, Ecole Nationale Supérieure Agronomique, Montpellier, Ecole doctorale : Biologie Intégrative, Discipline : Biologie, 270p.

Herrero M, Thornton PK, Notenbaert AM, Wood S, Msangi S, Freeman HA, Lynam J. 2010. Smart investments in sustainable food production: revisiting mixed crop-livestock systems. Sci., 327(5967) : 822-825.

Institut Nationale de la Statistique (INS). 2012. Recensement Général de la Population et l'Habitat (RGP/H). INS : Niger, 15.

Jamin JY, Havard M, Mbetid-Bessane E, Djamen Nana P, Djonnewa A, Djondang K, Leroy J. 2007. Modélisation de la diversité des exploitations. In Exploitations Agricoles Familiales en Afrique de l'Ouest et du Centre : Enjeux, Caractéristiques et Eléments de Gestion, ed. Qae ; 123-153.

Jouve P. 2007. Le jeu croisé des dynamiques agraires et foncières en Afrique subsaharienne. Cah. Agri., 16(5): 379385 DOI: 10.1684/agr.2007.0136.

Lawali S. 2011. Dynamique des transactions foncières et vulnérabilité rurale au Niger 
: cas des communes rurales de Tchadoua et Yaouri ; Thèse de doctorat ; Université de Liège ; Belgique, $266 \mathrm{p}$.

Mahamane A, Mahamane S, Yacoubou B, Issaka A, Ichaou A, Saley K. 2007. Analyse diachronique de l'occupation des terres et caractéristiques de la végétation dans la commune de Gabi (région de Maradi, Niger). Sécheresse. 18(4) : 296-304 DOI : 10.1684/sec.2007.0105.

Maidawa MH. 2013. Enjeux fonciers et stratégies des acteurs autour de la vallée de la Tarka dans le département de Dakoro (Maradi/Niger). Mémoire de Master II. Université Abdou Moumouni, Niamey, 68p.

Massouroudini A, Sébastien K, Mamadou S, Jean C, Jérémy B, Chantal KZ. 2016. Influence des activités agricoles sur la végétation le long d'un gradient pluviométrique nord-sud du Burkina Faso. VertigO., 16(1): DOI : 10.4000 /vertigo. 17015

Mbétid-Bessane E, Havard M, Djondang K. 2006. Evolution des pratiques de gestion dans les exploitations agricoles familiales des savanes cotonnières d'Afrique centrale. Cah. Agric., 15(6): 555-559. DOI: 10.1684/agr.2006.0038

Ministère de l'Elevage (MEL). 2015. Evolution du cheptel nigérien : Statistiques sur l'élevage au Niger, 76p.

Ministère de la Coopération. 1998. Production animale. Zootechnie In Mémento de l'Agronome, (4 $4^{\mathrm{è}}$ édn). CIRAD-GRET, 1119-1163.

R development Core Team. 2010. A Language and Environment for Statistical Computing. R Foundation for Statistical Computing: Vienna, Austria.

Rueff M. 2011. Vers une meilleure compréhension des systèmes d'exploitation pour un renforcement du conseil à l'exploitation familiale des organisations paysannes au Burkina Faso. Mémoire de fin d'étude, Ecole Nationale Supérieure Agronomique de Toulouse, http://catalog.ihsn.org/index. $\mathrm{php} /$ citations/44006. 